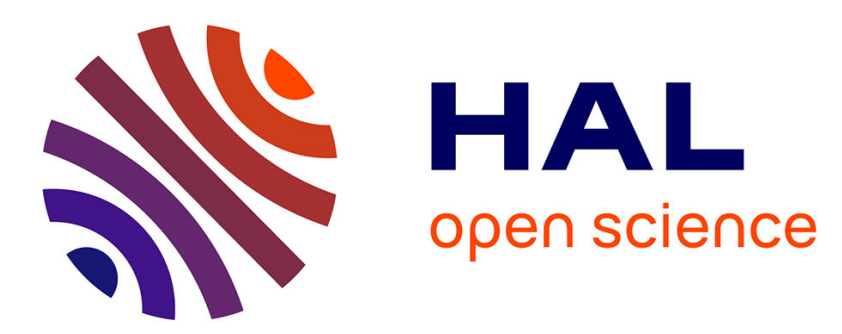

\title{
Impact du faciès sédimento-diagénétique sur l'altération en ouvre d'un géomatériau calcaire
}

\author{
Gilles Fronteau, Vincent Barbin, André Pascal
}

\section{To cite this version:}

Gilles Fronteau, Vincent Barbin, André Pascal. Impact du faciès sédimento-diagénétique sur l'altération en œuvre d'un géomatériau calcaire. Comptes Rendus de l'Académie des Sciences - Series IIA - Earth and Planetary Science, 1999, 328 (10), pp.671 - 677. 10.1016/S1251-8050(99)80176-1. hal-01694423

\section{HAL Id: hal-01694423 \\ https://hal.science/hal-01694423}

Submitted on 27 Jan 2018

HAL is a multi-disciplinary open access archive for the deposit and dissemination of scientific research documents, whether they are published or not. The documents may come from teaching and research institutions in France or abroad, or from public or private research centers.
L'archive ouverte pluridisciplinaire HAL, est destinée au dépôt et à la diffusion de documents scientifiques de niveau recherche, publiés ou non, émanant des établissements d'enseignement et de recherche français ou étrangers, des laboratoires publics ou privés. 


\title{
Impact of sedimentary-diagenetic facies on weathering of carbonate building stone
}

\section{Impact du faciès sédimento-diagénétique sur l'altération en œuvre d'un géomatériau calcaire}

Gilles Fronteau* Vincent Barbin, André Pascal

GEGENAA, EA3795, Université de Reims-Champagne-Ardenne, 2 esplanade Roland-Garros, 51100

Reims, France. gilles.fronteau@univ-reims.fr

\begin{abstract}
:
Observation and characterization of weathering of different buildings stones from the eastern area of the Paris Basin show the importance of sedimentary and diagenetic facies. A comparison between the harshness of environment exposure and the microfacies allows the evolution of stone decay to be classified according to different telogenetic behaviours. The durability of building stones can be related to their conditions of use, to the developed weathering or to the degradation conditioned by the microfacies of the limestone ('preferential weathering').
\end{abstract}

Keywords: weathering / monument / limestone / diagenesis / Paris Basin / France

\section{Résumé :}

L'observation et la caractérisation de l'altération en oeuvre de différentes roches de l'est du Bassin de Paris font apparaître l'importance du faciès sédimento-diagénétique pour expliquer leur dégradation. La comparaison de l'agressivité du milieu d'exposition avec le microfaciès permet de classer les évolutions en œuvre selon différents comportements télogénétiques. La durabilité de la pierre à bâtir peut alors s'exprimer, non seulement en fonction des conditions d'utilisation et de l'altération développée, mais également en fonction de la dégradation induite et contrôlée par le microfaciès de la roche (" altération préférentielle »).

Mots-clefs : altération / monument / calcaire / microfaciès / diagenèse / Bassin parisien / France

\section{Abridged version}

\section{Introduction}

Since the nineteen seventies, restoration of monuments has been accompanied by studies of rock weathering (Winkler, 1973; Jeannette, 1981; Camuffo et al., 1982). Different aspects of rock decay such as the typical alteration pathway, the morphology or the growth and/or dissolution of components have been studied (Galan, 1989; Viles, 1990). However, the influence of these alteration processes on the stone itself are poorly known.

The complexity of the reactions and the large numbers of variables do not allow results from a specific monument to be generalized (Quénée, 1990).

Weathering of building stone begins when the rock enters the last diagenetic stage of a sediment, the telogenetic realm during uplift (Choquette and Pray, 1970). There, the physicochemical conditions 
Fronteau G., Barbin V., Pascal A., 1999, Impact of sedimentary-diagenetic facies on weathering of carbonate building stone. Comptes Rendus de I'Académie des Sciences - Series IIA - Earth and Planetary Science, Volume 328, Issue 10, May 1999, p. 671-677. doi:10.1016/S1251-8050(99)80176-1

continuously change and thus alteration of the rock will occur depending on the environment and the resistance of the rock against weathering. Studies dealing with the weathering behaviour of rocks must therefore take into account the physico-chemical conditions of the environment and the resistance of the rock to weathering. Studies by Bell (1993) and Vleugles et al. (1995) focused on the effect of the microclimate on stone weathering by studying the influence of parameters such as porosity, compressive strength or chemical composition of the rock. The effect of textural variables, e.g. type of grains or cement, was not taken into account.

In this study we aim to show the importance of the sedimentary/diagenetic microfacies with respect to the weathering potential of a limestone. Knowledge of this microfacies contributes to a better understanding of the weathering stability of limestones used in buildings.

\section{Relationship between microclimate and weathering}

Sansom-Gombert (1991) and Philippon et al. (1992) clearly demonstrated the close relationship between rock weathering and microclimate, i.e. exposure of the surface, porewater composition and atmospheric conditions.

The exposure of a limestone to rain favours dissolution at the surface and rainwater penetration into the surface rim of the rock. In areas protected from rain, atmospheric dust particles may accumulate resulting in a black crust or calcium salts may crystallize as white crusts. In wet zones surface dissolution can produce crusts exceeding one centimetre in thickness (Del Monte and Furlan, 1995). Air pollution greatly enhances the effect of surface alteration through acidification of the rainwater (Allesandrini et al., 1992), deposition of vanadium-rich fly-ashes (Ausset et al., 1992), or changes in the fungal population on the surface of buildings (Krumbein and Gorbushina, 1994). However, the relationships between exposure and weathering do not explain all weathering aspects observed in building stones. Indeed, different rock types exposed to identical weathering conditions may alter differently. In these cases, rock properties result in different responses of the rock to weathering.

\section{Sampled material and methodology}

A suite of characteristic limestones used for buildings in the Champagne-Ardenne region were selected for this study. Fresh samples were taken in the original quarries (figure 1) to determine the variability of the microfacies and to characterize the original rock type. Weathered samples were obtained from several cities in the Champagne-Ardenne and surrounding regions.

Quantitative analyses of the porosity, the different types of particles and cements were performed with the image analysis software of Saisam and Areas (Microvisions Instruments). Optical microscopy, cathodoluminescence microscopy (Barbin and Shvoerer, 1997) and epifluorescence microscopy (Dravis and Yukewicz, 1985) were used to characterize microfacies and diagenetic sequences of the limestones. The microscopes used were an Olympus BX-50 equipped with a cold cathode unit from OPEA and an Olympus BX-60 with epifluoresence adapter and a cube of excitation type U-MVU (330$385 \mathrm{~nm})$.

\section{Relationship microfacies-weathering}

In order to classify the observed weathering phenomena, the following morphological features are used: (1) crusts and patinas formed on the surface of the rock (Arnold and Zehnder, 1989); (2) dissolution (hollows or scourings); (3) desquamation (lamellar or tabular spalling, exfoliation); (4) disintegration (loss of components, cellular decay (Pauly, 1996); (5) fragmentation (partial loss due to structural heterogeneities, e.g. bedding, stylolites). 
Fronteau G., Barbin V., Pascal A., 1999, Impact of sedimentary-diagenetic facies on weathering of carbonate building stone. Comptes Rendus de l'Académie des Sciences - Series IIA - Earth and Planetary Science, Volume 328, Issue 10, May 1999, p. 671-677. doi:10.1016/S1251-8050(99)80176-1

Limestones from Savonniéres and Dom preferentially undergo a sandy disintegration (cellular decay), These rocks are porous, poorly cemented by sparry calcite and show a granular microstructure. The disintegration of the limestone from Savonnieres affects more or less homogeneously the entire rock surface. In contrast, the disintegration of the limestone from Dom is heterogeneous with deeper eroded layers of a slightly different microfacies on the rock surface.

Micritic limestones such as those from Courville, Bar-surAube and Lutetian limestones with Ditrupa (serpulid worms) develop thin, $\mathrm{mm}$-thick layers of desquamation. The addition of other particles in the micritic limestone, e.g. quartz grains (Lutetian limestone in Ditrupa facies), generates a crumbly rock which is very sensitive to disintegration.

Strongly cemented granular limestones such as those of Euville and Dijon show an intermediate rate of weathering with desquamation resulting in loss of cohesion and disintegration of components.

Limestones with a heterogeneous structure such as those from Dijon with bedding planes and/or stylolites and the Blue stone of Givet which contains calcite veins are characterized by fragmentation and loss of loose parts.

\section{Preferential weathering and evolution of weathering}

Although the evolution of weathering is determined by the microclimate during exposure, weathering may vary as a function of the sedimentary/diagenetic microfacies. The effect of the microfacies is most prominent when the local conditions are less severe, e.g. in rural regions or on flat walls. Under aggressive chemical conditions, e.g. polluted urban regions, strong stone decay develops and overprints the saddle effects of the microfacies. The relationship between limestone microfacies and aggressivity of the microclimate allows to postulate the concept of telogenetic resistivity (figure 2 ). This resistivity of the rock directly controls the persistency against the local microclimate and thus the durability as a building material. Each limestone and mircrofacies may thus evolve on a different path, e.g. rapid destruction, decay after preferential weathering or decay depending on the microclimate.

\section{Telogenetic resistivity of building stones}

Rocks with a weak resistance against weathering disintegrate rapidly and thus show a type A resistivity, e.g. 'unsuitable stone'.

Under sensitive architectural conditions, e.g. carvings, capillary zones, the aggressivity of the microclimate may be focused and thus also weathering. For example, the Stone of Courville shows desquamation and erosion of the limestone. This persistency against weathering is typical of type B resistivity, e.g. 'inappropriate stone'.

Type $C$ and $D$ resistivities correspond to an aggressive environment. Type $C$, 'damaged stone', is typical for conditions where the microclimate enhances rock weathering. In the case of type $D$, 'altered stone', weathering is solely controlled by the microclimate and not affected by the microfacies.

In cases where the agressivity of the microclimate has no strong influence on the rock, weathering is a slow process (type E resistivity or 'Preserved rock') and adopts the morphology induced by the microfacies (cellular decay for granular rocks, desquamation or dissolution for micritic limestones).

Type $\mathrm{F}$ resistivity is hypothetical as this rock is persistent against any agressive microclimate and thus does not alter ('kept stone').

\section{Conclusions}


A new approach to the description of building stone weathering is proposed based on the sedimentary/diagenetic microfacies of the limestone and the microclimate conditions during rock exposure. This new approach allows prediction of weathering behaviour of building stone in the future. A detailed analysis of the microfacies including the quantification of porosity, cementation and components (image analysis) is necessary to characterize the weathering behaviour of a limestone. Both the effects of microfacies and microclimate on limestone weathering are distinguishable and thus the most favourable limestone with the highest weathering resistivity for a given application can be determined. In addition, knowledge of the weathering resistivity of a specific limestone may help to decide whether restoration or replacement is the adequate method.

\section{Article (in French)}

\section{Introduction}

La dégradation des constructions en Pierre calcaire est préoccupante. Dès les années soixante-dix, les restaurations de monuments ont été accompagnées de l'étude des altérations en œuvre (Winkler, 1 973 ; Jeannette, 1981, Camuffo et al., 1 982). Les " maladies de la Pierre " ont été décrites : caractéristiques de leur développement, morphologie, taux de croissance ou de dissolution (Galan, 1 989 ; Viles, 1 990). Cependant, l'impact de ces transformations sur le matériau lui-même reste mal connu. De plus, la complexité des mécanismes et le grand nombre de facteurs intervenants rendent difficile la généralisation de ces résultats (Quénée, 1990 ).

L'altération en œuvre d'une roche calcaire peut être assimilée aux ultimes étapes diagénétiques de l'évolution du sédiment (télogenèse : Choquette et Pray, 1970) conduisant ä sa destruction (et ä son recyclage). La roche, au contact de l'atmosphère, subit une rupture de l'équilibre acquis fors de son induration et de son enfouissement. Ce déséquilibre correspond ä la différence entre l'agressivité de l'environnement et la résistance de la roche. L'étude de l'altération d'un géomatériau doit donc prendre en compte ces deux pôles de paramètres, afin de les confronter. Les travaux antérieurs (Bell, 1993 ; Vleugels et al., $1995 \ldots$..) ont axé leur problématique sur la caractérisation du microclimat d'exposition, en ramenant l'influence de la roche aux seuls caractères physiques (porosité globale, résistance ä la compression), ou sa composition chimique globale... L 'effet de la composition de la roche (nature du liant - ciments ou matrices des grains, de la texture ou de la structure sédimentaire - litages, stylolithes, etc.) par rapport ä l'impact du microclimat d'exposition des blocs en oeuvre n'est pas pris en compte.

Nous mettrons en évidence ici l'importance du microfaciès (sédimentaire ou diagénétique) dans l'altération d'une roche calcaire et montrerons comment sa caractérisation permet de mieux comprendre son comportement en oeuvre.

\section{Les « maladies » de la Pierre}

Les dégradations en œuvre ( « maladies de la Pierre ») sont classées de deux façons : selon les différents processus d'altération (chimiques, physiques, biologiques...) ou selon la morphologie des dégradations. Le second type de classification est plus approprié, car les interactions entre les divers processus d'altération sont trop importantes pour être séparées : par exemple, les desquamations dues ä la fissuration de la roche sont principalement causées par la pression de cristallisation du gypse (processus chimique) (Arnold et Zehnder, 1 989) ou ä la fréquence des cycles d'hydratationdéshydratation des sels " gonflants » (processus physique) (Pauly, 1 996). 
Nous utiliserons donc une classification des altérations en fonction de leur morphologie (tableau 1) : 1 : croûtes et patines se formant ä la surface des blocs ; 2 : dissolutions : creusement ou ravinement de la roche ; 3 : desquamations : débit en mille-feuilles ou en plaques, exfoliations ; $4:$ désagrégation : perte d'éléments ; 5 : fragmentation : perte d'une partie d'un bloc liée à des hétérogénéités de structure.

Notons que, pour limiter le nombre de variables, cette étude ne prend pas en compte les altérations liées une incompatibilité entre les blocs de calcaires et le liant de maçonnerie (mortier ou ciment), liant qui peut modifier la réponse des calcaires à l'altération et donc influencer le comportement télogénétique de la roche (barrière de porosité, contamination chimique, etc.).

Tableau. Modes d'altération en œuvre des calcaires étudiés.

Weathering types of different French building stones.

\begin{tabular}{|c|c|c|c|c|c|c|}
\hline & Nom de la roche & 1 : encroûtement & 2 : dissolution & $3:$ desquamation & 4 : désagrégation & 5 : fragmentation \\
\hline 1 & Pierre de Savonnières & + & + & / & +++ & / \\
\hline 2 & Pierre de Dom & ++ & + & ++ & +++ & + \\
\hline 3 & Calcaire d'Euville & + & + & ++ & ++ & / \\
\hline 4 & Pierre de Dijnn & ++ & + & ++ & ++ & +++ \\
\hline 5 & Pierre noire de Givet & + & + & ++ & + & +++ \\
\hline 6 & Liais de Courville & ++ & ++ & +++ & $/$ & I \\
\hline 7 & Pierre de Bar-sur-Aube & +++ & ++ & +++ & + & / \\
\hline 8 & Calcaire à Ditrupa & ++ & + & ++ & ++ & + \\
\hline
\end{tabular}

/: altération rarement observée (moins de $5 \%$ des cas).

+ : altération parfois observée ( $5-25 \%$ des cas).

++ : altération souvent observée (25-50\% des cas).

+++ : altération observée dans la plupart des cas (plus de $50 \%$ des cas).

/: weathering rarely observed (less than $5 \%$ ).

+ : weathering sometimes observed $(5-25 \%)$

++ : weathering often observed $(25-50 \%)$.

+++ ; weathering usually observed (more than $50 \%$ ).

\section{Relations microclimat d'exposition — altération}

L'étroite relation entre l'altération développée en un point et le microclimat environnant est démontrée (Philippon et al., 1992) ; la dégradation des éléments d'une construction est liée à leurs conditions d'exposition. Ainsi, selon Sansom-Combert (1991), la répartition, l'apparition et la croissance des altérations sur une roche donnée dépendent majoritairement de l'exposition du bloc, des circulations d'eau et de la composition de l'atmosphère ambiante.

L'exposition aux pluies battantes favorise les dissolutions de surface et le ravinement, le ruissellement érode les zones externes de la roche. Dans les zones abritées, les particules d'origine atmosphérique s'accumulent, les sels de calcium cristallisent en surface ou peuvent épigéniser la calcite ; des croûtes noires (cendres volantes), des croûtes blanches (recristallisation de calcite) se développent. Dans les zones humides à la périphérie des zones lessivées par la pluie, les croûtes peuvent dépasser un centimètre d'épaisseur (Del Monte et Furlan, 1 995).

Aux endroits soumis à de fréquents cycles hydratation - déshydratation (ou subissant de fortes variations thermiques), les blocs sont fissurés ou desquamés. Ces dégradations sont dues au gel ou aux 
Fronteau G., Barbin V., Pascal A., 1999, Impact of sedimentary-diagenetic facies on weathering of carbonate building stone. Comptes Rendus de l'Académie des Sciences - Series IIA - Earth and Planetary Science, Volume 328, Issue 10, May 1999, p. 671-677. doi:10.1016/S1251-8050(99)80176-1

sels, qui gonflent lors de leur hydratation ou cristallisent lors de l'évaporation de l'eau et font éclater la roche.

La pollution tend à amplifier ces dégradations, par exemple en augmentant la concentration des composés chimiques impliqués : acidification de l'eau (Allessandrini, et al., 1992), dépôt de particules qui catalysent les réactions, telles que les cendres volantes contenant du vanadium (Ausset et al., 1992), ou bien en modifiant les populations d'organismes à la surface des bâtiments (Krumbein et Corbushina, 1994).

Les relations microclimat d'exposition - altérations n'expliquent pas la totalité du comportement en oeuvre des roches. En effet, dans des conditions identiques, les roches peuvent présenter des dégradations différentes ; les caractéristiques propres aux géomatériaux contrôlent donc leur altérabilité, en modifiant leur réponse à l'agressivité du milieu.

\section{Sites d'échantillonnage}

Nous avons sélectionné plusieurs pierres à bâtir en raison de leur représentativité des calcaires utilisés en construction dans la région Champagne-Ardenne (voir figure 1). Elles sont issues de formations sédimentaires d'âges très différents - du Givétien au Lutétien.

L'échantillonnage des sites originaux d'extraction permet de caractériser la roche saine et de déterminer la variabilité du microfaciès dans la formation. Les échantillons altérés en œuvre proviennent de différentes villes de Champagne-Ardenne et des régions environnantes.

\section{Méthodes d'étude des microfaciès}

L'analyse sédimento-diagénétique, pratiquée sur les échantillons prélevés en carrière, met en évidence les composants sédimentaires et diagénétiques qui contrôlent de nombreuses propriétés de la roche : types des grains et de ciments, importance et morphologie de la porosité... Cette dernière et les différentes proportions d'éléments ont été mesurées par analyse d'images (logiciels Saisam et Aréas de Microvisions Instruments).

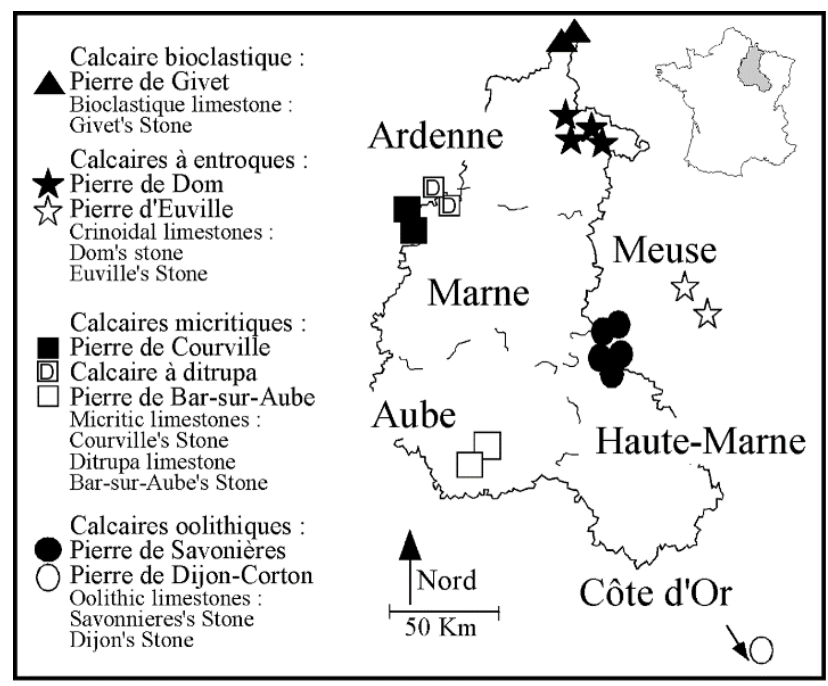

Figure 1. Calcaires étudiés : localisation et faciès principal.

Figure 1. Studied limestones: location and facies. 
Fronteau G., Barbin V., Pascal A., 1999, Impact of sedimentary-diagenetic facies on weathering of carbonate building stone. Comptes Rendus de I'Académie des Sciences - Series IIA - Earth and Planetary Science, Volume 328, Issue 10, May 1999, p. 671-677. doi:10.1016/S1251-8050(99)80176-1

Pour affiner le séquençage diagénétique (Durlet et al., 1 992) et la caractérisation des calcaires sains, I'utilisation du microscope optique polarisant a été associée à la microscopie en cathodoluminescence (Barbin et Shvoerer, 1997) et à fa microscopie en épifluorescence (Dravis et Yukewicz, 1 985). Les microscopes utilisés sont de type Olympus BX-50, équipé d'une cathode froide OPEA et BX-60, avec un cube d'excitation de type U-MWU (longueur d'onde de la fluorescence UV).

\section{Relation microfaciès-altération}

En classant les altérations observées sur divers calcaires, se côtoyant souvent sur une même construction, nous proposons les éléments présentés dans le tableau (qui ne tient pas compte de la notion de durabilité).

Les observations ont été réalisées en de nombreux points, depuis le Nord des Ardennes jusqu'à la région dijonnaise, aussi bien sur des monuments historiques en milieu urbain que sur de simples habitations en zone rurale. Ces résultats regroupent ainsi divers types et degrés de pol lutions, de climats, de positions architecturales et de durées de mises en œuvre. En rapportant les altérations observées au microfaciès du calcaire, on dégage les grandes lignes du vieillissement de ces roches.

Les calcaires subissant préférentiellement une désagrégation sableuse (alvéolisation) comme la pierre de Savonnières (1) et la pierre de Dom (2), ont un faciès granulaire faiblement cimenté (le liant sparitique n'obture pas la porosité intergranulaire). La désagrégation de la pierre de Savonnières (1) affecte de grandes surfaces et la plupart des litages obliques. En revanche, la désagrégation de la pierre de Dom affecte surtout certains litages, dont le microfaciès varie légèrement; ces litages sont surcreusés par rapport au reste du bloc, uniquement altéré en surface.

À l'opposé, les calcaires à phase matricielle micritique (comme le Liais de Courville (6), la pierre de Barsur-Aube (7) et le calcaire à Ditrupa (8) développent surtout des desquamations en feuillets d'épaisseurs millimétriques. Cependant, l'augmentation du nombre d'éléments figurés (comme les grains de quartz dans le calcaire lutétien à Ditrupa) rend la roche plus friable ; sa sensibilité à la désagrégation augmente largement.

Les calcaires granulaires fortement cimentés (pierre d'Euville (3) et pierre de Dijon (4) présentent des modes d'altération intermédiaires : la roche se desquame en épais feuillets ou bien en plaques (exfoliation), puis, la cohésion du calcaire étant moindre, les éléments sont dissociés (désagrégation). Dans le cas de la pierre d'Euville (3), la grande taille des éléments conduit à la formation d'une plaque épaisse, qui se décolle puis se fragmente rapidement. Alors que, pour la pierre de Dijon (4), la cimentation lie fortement oolithes et bioclastes, la perméabilité plus faible limite la desquamation à la surface des blocs. Les alvéoles, résultats de la désagrégation, ne se développent qu'ensuite, dans l'épaisseur de roche déjà dégradée.

Certains calcaires ont une structure hétérogène, comme la pierre de Dijon (4), avec des litages obliques très marqués ou des stylolithes d'origine sédimentaire et la pierre bleue de Givet (5), présentant des veines de calcite sparitique. Leur altération préférentielle est une fragmentation en fonction de ces hétérogénéités, qui forment des barrières de porosité ou des surfaces de faiblesses structurales.

\section{Notions « d'altération préférentielle » et « d'altération développée »}

Si le développement des altérations est conditionné par le microclimat d'exposition du bloc, ce tableau montre que, pour un microfaciès donné, il existe un type d'altération préférentielle. Cette altération se développera dans des conditions d'environnement naturel ou peu agressif (en milieu rural ou au centre des élévations de murs). Alors que, dans des conditions plus agressives (en milieu urbain pollué 
Fronteau G., Barbin V., Pascal A., 1999, Impact of sedimentary-diagenetic facies on weathering of carbonate building stone. Comptes Rendus de I'Académie des Sciences - Series IIA - Earth and Planetary Science, Volume 328, Issue 10, May 1999, p. 671-677. doi:10.1016/S1251-8050(99)80176-1

par exemple), se développera une altération liée à l'environnement, masquant le comportement induit par le microfaciès.

Cette relation entre la composition faciologique de la roche et l'agressivité du milieu d'exposition nous conduit à proposer la notion de " comportement télogénétique " (ou comportement en œuvre) (voir figure 2). La durabilité en œuvre (différence entre l'agressivité du milieu et la résistance de ta pierre) est fonction du comportement télogénétique adopté, avec, dans certains cas, amplification de I'altération. Ainsi, chaque pierre à bâtir (et même chaque variété de microfaciès) peut évoluer selon divers modèles d'altérations : destruction rapide, dégradation suivant l'altération préférentielle ou développement d'une altération liée au milieu d'exposition.

\section{Comportements télogénétiques d'une roche en œuvre (figure 2):}

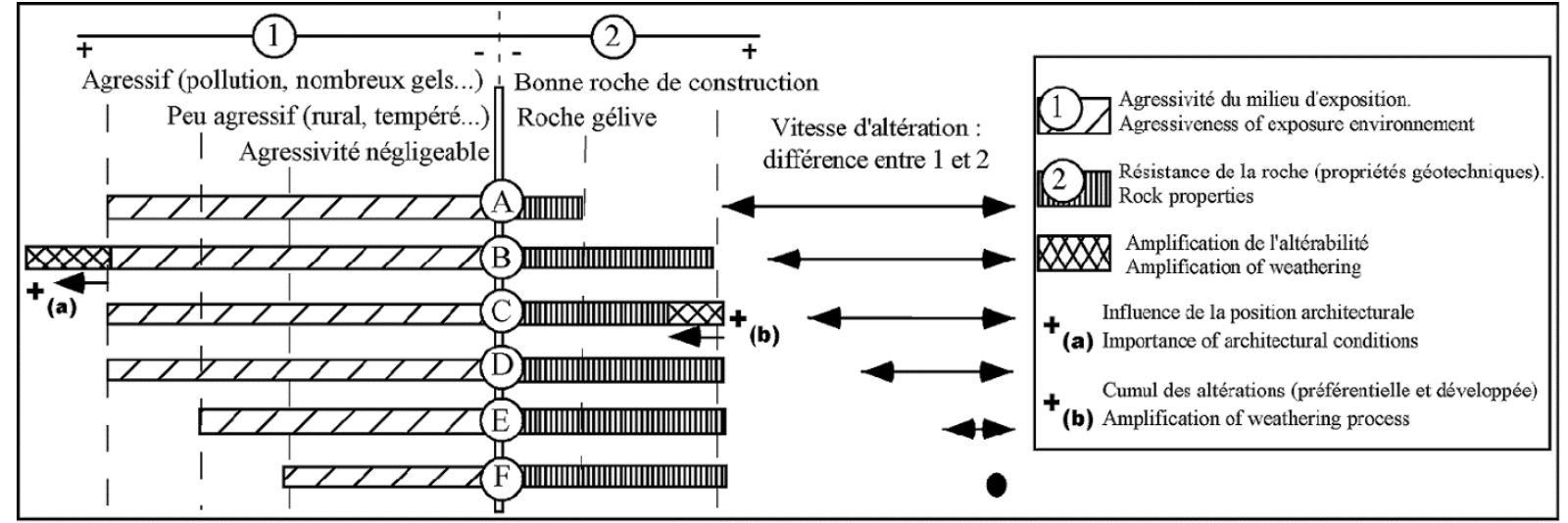

A Pierre inapte : ne résiste pas aux contraintes liées à une utilisation en oeuvre

A Inapt stone : don't resist if use like building stone

B Pierre inadaptée : certaines conditions d'utilisations provoquent la destruction de la roche

Inappropriate stone : specific architectural conditions

(C) Pierre agressée : l'altération developpée correspond à l'altération préférentielle de la roche : amplification

C) Damaged stone : develloped weathering corespond to the preferential weathering of the faciès : amplification

(D) Pierre altérée : l'utilisation en oeuvre de la roche entraîne une altération différente de l'altération préférentielle.

D) Altered stone : develloped weathering different to the preferential weathering of the faciès

(E) Pierre préservée : 1e milieu d'utilisation est peu agressif, la roche s'altére lentement selon son altération préférentielle

E Preserved stone : in a environment poorly agressive, slow weathering according preferential weathering of the facies

(F) Pierre conservée : la rupture d'équilibre liée à l'utilisation en oeuvre est faible, la roche ne ne dégrade pas.

Kept stone : there is no imbalance between agressiveness of the environment and rock properties : no weathering

Figure 2. Comportements télogénétiques théoriques d'une roche en œuvre.

Telogenetic resistivity of building stones.

Inapt stone: not resistant if used as a building stone.

Pierre inapte : ne résiste pas aux contraintes liées à une utilisation en œuvre.

Inappropriate stone: specific architectural conditions.

Pierre inadaptée : certaines conditions d'utilisation provoquent la destruction de la roche.

Damaged stone: developed weathering corresponds to the preferential weathering of the facies: amplification.

Pierre agressée : l'altération développée correspond à l'altération préférentielle de la roche : amplification. 
Fronteau G., Barbin V., Pascal A., 1999, Impact of sedimentary-diagenetic facies on weathering of carbonate building stone. Comptes Rendus de l'Académie des Sciences - Series IIA - Earth and Planetary Science, Volume 328, Issue 10, May 1999, p. 671-677. doi:10.1016/S1251-8050(99)80176-1

Altered stone: developed weathering different from the preferential weathering of the facies.

Pierre altérée : I'utilisation en œuvre de la roche entraîne une altération différente de l'altération préférentielle.

Preserved stone: in an unaggressive environment, slow weathering, according to preferential weathering of the facies.

Pierre préservée : le milieu d'utilisation est peu agressif, la roche s'altère lentement, selon son altération préférentielle.

Kept (protected) stone: there is no unbalance between aggressiveness of the environment and rock properties: no weathering.

Pierre conservée : la rupture d'équilibre liée à l'utilisation en œuvre est faible, la roche ne se dégrade pas.

Certaines roches sont inaptes à l'utilisation en construction : elles sont gélives ou ont une résistance à la compression insuffisante. Elles sont rapidement dégradées et détruites et présentent un comportement de type A : " pierre inapte ». Dans le comportement de type B : " pierre inadaptée », l'agression du milieu est fortement amplifiée par les conditions de mise en oeuvre (nombreux angles, remontées capillaires...). De nombreuses roches ont ainsi des limitations d'utilisation : ainsi, par exemple, l'utilisation en rebord de fenêtre de la pierre de Courville entraîne une desquamation intense et l'érosion du bloc.

Les comportements $C$ et $D$ correspondent à une utilisation normale dans un milieu agressif : milieu urbain par exemple. Dans le type C (« pierre dégradée »), l'altération qui se développe du fait du milieu correspond à l'altération préférentielle de la roche, et il se produit un phénomène d'amplification. Dans le type $D$ ( " pierre altérée »), l'altération développée diffère ou s'oppose au mode de dégradation lié au microfaciès, la roche s'altère selon un mode conditionné par le milieu. Par exemple, la pierre d'Euville, utilisée pour les soubassements de nombreux édifices, possède une durabilité importante (comportements de types D ou E). Mais, aux angles des blocs, la desquamation provoque une diminution de la cohésion de la roche, qui se désagrège alors rapidement, le cumul de l'altération et de l'altérabilité provoquant la dégradation de la roche (type $\mathrm{C}$ ).

Quand l'agressivité du milieu n'affecte pas particulièrement la roche, l'altération se développe lentement (de type $E$ : « pierre préservée »), selon l'altération préférentielle conditionnée par le microfaciès (désagrégation pour les calcaires granulaires, desquamation en cloques et dissolution pour les calcaires micritiques...). Enfin, le type $\mathrm{F}$ est un cas hypothétique où la roche ne subit pas d'agression particulière et ne s'altère donc pas (« pierre conser-

\section{Conclusions}

Une nouvelle approche de l'étude des altérations en œuvre est proposée. II ne s'agit plus de se limiter à la morphologie et à la vitesse de développement des dégradations, mais, en analysant l'évolution du calcaire luimême, d'établir des relations entre l'altération en œuvre et le faciès sédimentodiagénétique. Cette approche permet de déterminer des types prévisibles de comportements.

Ces relations, qui peuvent être considérées comme simples, en système général de cause/effet (un calcaire granulaire se désagrège, un calcaire micritique se desquame), sont en fait complexes dans le 
Fronteau G., Barbin V., Pascal A., 1999, Impact of sedimentary-diagenetic facies on weathering of carbonate building stone. Comptes Rendus de I'Académie des Sciences - Series IIA - Earth and Planetary Science, Volume 328, Issue 10, May 1999, p. 671-677. doi:10.1016/S1251-8050(99)80176-1

détail et à l'échelle du bloc de roche mis en oeuvre. Des variations faciologiques dans le taux de cimentation, la proportion des éléments... entraînent des modifications du comportement télogénétique. La caractérisation de l'altération d'un calcaire doit donc comporter une analyse fine de son microfaciés sédimento-diagénétique, dans laquelle seront quantifiés la porosité, la cimentation et les divers éléments figurés (quartz, glauconie, oolithes, bioclastes, etc.).

La prise en compte et la mise en relation du faciès d'une roche calcaire et de son altération en oeuvre permettent de hiérarchiser son altérabilité. II est possible de distinguer les effets de "l'altération privilégiée " (induite et contrôlée par le microfaciès) et de " l'altération développée " (conditionnée par le microclimat d'exposition), pour utiliser au mieux une roche calcaire, prévoir sa durabilité et éviter le cumul des facteurs défavorables. Dans l'idéal, l'étude du comportement en oeuvre d'un calcaire devrait donc inclure l'analyse de la roche, de l'environnement d'exposition mais aussi des conditions de mise en oeuvre (notamment l'influence du mortier ou du liant de maçonnerie). La définition de comportements télogénétiques possibles pour une pierre, avec la détermination des paramètres internes et externes qui les conditionnent, doit permettre de mieux cerner l'évolution en oeuvre des géomatériaux et d'intervenir efficacement dans le choix des traitements de restauration ou de pierres de substitution.

\section{Références}

Allessandrini et al. 1 992. Stone materials of Noto (Syracusa) and their decay, in: 7th International Congress on Deterioration and Conservation ofStone: proceedings, Lisbonne, $1,11-20$

Arnold A. et Zehnder K. 1990. Salt weathering on monuments, in: Veniale F. et Zezza F. (éds), Proc. Symp. Analytical methodologies for the investigations of damaged stones, Pavia, $31-57$

Ausset $P$. et al. 1991 . Large scale distribution of fly-ash particles inside weathering crust on calcium carbonate substrates: some examples on French monuments, in: 2 nd International symposium on the conservation of monuments in the Mediterranean Basin, Geneva,

Barbin V. et Schvoerer M. 1997. Cathodoluminescence et géosciences, C R. Acad. Sci. Paris, 325, série 'la, 1 57169

Bell F.G. 1 993. Durability of carbonate rock as building stone with comments on its preservation, Environ. Geol., $21,187-200$

Camuffo D. et al. 1982 . Origin and growth mechanisms of the sulfated crusts on urban limestone, Water Air Soil Pollut., 19 , 351-359

Choquette P.W. et Pray L.C. 1 970. Geologic nomenclature and classification of porosity in sedimentary carbonates, Bull. AAPG, 54 (2), 207-250

Del Monte M. et Furlan V. 1 995. Cr00tes noires, sulfatation et dégradation de la Pierre, in : Proceedings ofLCP, 7995, Conservation et restauration de biens culturels, Montreux, 349-369.

Dravis J.J. et Yukewicz D.A. 1985 . Enhanced carbonate petrography using fluorescence microscopy, J. Sediment. Petrol., 55, 6, 795-804

Durlet C. , Loreau J.-P. et Pascal A. 1992. Signature diagénétique des discontinuités et nouvelle représentation de la diagenése, C. R. Acad. Sci. Paris, 31 4, série II, 1 507-1 514

Galan E. 1 989. Carbonate rocks: alteration and control of stone quality, some considerations, in : Zezza F. (éd.), 7 st International Symposium on the conservation of monuments in the Mediterranean Basin, Bari, 249-254

Jeannette D. 1981 . Modifications superficietles de gres en oeuvre dans des monuments alsaciens, Sci. Géol. Bull., 34 (1), 37-46

Krumbein W.E. et Gorbushina A.A. 1994. Organic pollution and rock decay, in : 3th International Symposium on the conservation of monuments in the Mediterranean Basin, Venise, 277-284

Pauly J.-P. 1996. Altération alvéolaire : évolution et climatologie, Mém. Soc. géol. France, $169,421-426$

Philippon J. et al. 1 992. La conservation de la Pierre monumentale en France, Presses du CNRS, 320 p. 
Fronteau G., Barbin V., Pascal A., 1999, Impact of sedimentary-diagenetic facies on weathering of carbonate building stone. Comptes Rendus de l'Académie des Sciences - Series IIA - Earth and Planetary Science, Volume 328, Issue 10, May 1999, p. 671-677. doi:10.1016/S1251-8050(99)80176-1

Quénée B. 1 990. Transformations minéralogiques et texturales de matériaux rocheux, mortiers et bétons d'ouvrage variés : approche de la cinétique des mécanismes et identification des facteurs responsables, Thése, Université de Nancy, 192 p. (inédit)

Samsom-Gombert C. 1993. Influences d'un environnement urbain et maritime sur les altérations d'un calcaire en oeuvre : la Pierre de Caen, Thése, Université de Caen, 298 p. (inédit)

Viles H.A. 1 990. The early stage of building stone decay in an urban environment, Atmos. Environ. A-Gen, 24 (1 229-232

Vleugels G. et al. 1 995. Surface composition alteration of bare and treated limestones after ambient exposure, Sci. Total Environ., 167, 59-69

Winkler E.M. 1 973. Stone: properties, durability in man's environment, Springer-Verlag, 230 p. 